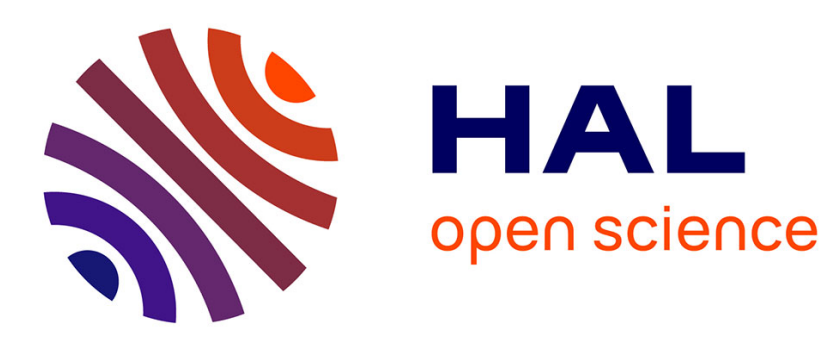

\title{
Modèle de prévision acoustique en espace clos basé sur les hypothèses de réflexion diffuse et spéculaire en parois
}

\author{
J. Delhom, J. Kyncl, M. Sram, C. Legros
}

\section{To cite this version:}

J. Delhom, J. Kyncl, M. Sram, C. Legros. Modèle de prévision acoustique en espace clos basé sur les hypothèses de réflexion diffuse et spéculaire en parois. Journal de Physique IV Proceedings, 1994, 04 (C5), pp.C5-117-C5-120. 10.1051/jp4:1994519 . jpa-00252872

\section{HAL Id: jpa-00252872 https://hal.science/jpa-00252872}

Submitted on 1 Jan 1994

HAL is a multi-disciplinary open access archive for the deposit and dissemination of scientific research documents, whether they are published or not. The documents may come from teaching and research institutions in France or abroad, or from public or private research centers.
L'archive ouverte pluridisciplinaire HAL, est destinée au dépôt et à la diffusion de documents scientifiques de niveau recherche, publiés ou non, émanant des établissements d'enseignement et de recherche français ou étrangers, des laboratoires publics ou privés. 


\title{
Modèle de prévision acoustique en espace clos basé sur les hypothèses de réflexion diffuse et spéculaire en parois
}

\author{
J.P. DELHOM, J. KYNCL, M. SRAM et C. LEGROS ${ }^{*}$
}

VUZORT a.s., Plzenska 66, 15124 Prague 5, Czech Republic

* Laboratoire d'Acoustique, Université de Toulouse Le Mirail, 5 allée Antonio Machado, 31058 Toulouse cedex, France

\begin{abstract}
This paper presents a mathematical model for the calculating of sound fields in enclosed areas, even where the internal dimensions are disproportionate : particular reference is made to the diffusive effect of walls. The model is based on a classical ray tracing method. However, not all of the reflected energy necessarily follows the specular path ; some of it can be scattered, forming, as it were new sources. The coefficient of diffusion associated with the sample of wall involved in the reflection regulates the sharing of the reflected energy. In order to determine this diffusion coefficient, we have developed a method that allows the measuring of the diffusion of a sample of wall. We have measured the sound propagation curves in several factory halls and compared the results to the previously calculated values. The high degree of correlation between the theoretical and experimental results validates the model and our approach of the coefficient of diffusion.
\end{abstract}

La plupart des modèles mathématiques développés jusqu'à présent pour prévoir le champ sonore en espace clos sont basés soit sur l'hypothèse de réflexion diffuse en parois [1], soit sur l'hypothèse de réflexion spéculaire. Il s'agit dans les deux cas d'hypothèses idéalistes qui n'existent pas dans la réalité où l'on se situe entre ces deux extrêmes [2]. L'avantage des modèles basés sur l'hypothèse de réflexion spéculaire (méthode des rayons ou sources-images) est qu'ils permettent le calcul aisé des paramètres temporels liés au champ sonore. Cependant dans les locaux à géométrie disproportionnée (c'est le cas de la plupart des halls de production industrielle), l'influence de la diffusion du son par les parois ne peut pas être négligée [3]. En effet, à grande distance de la source (à partir de 20 ou $30 \mathrm{~m}$ suivant les locaux), le phénomène de diffusion se traduit par un affaiblissement du niveau sonore s'accentuant avec la distance. Lorsque la diffusion n'est pas prise en compte, l'erreur sur le niveau sonore peut atteindre ou dépasser $10 \mathrm{~dB}$ à $100 \mathrm{~m}$. Cet article présente d'une part, un modèle informatique de prévision du champ acoustique dans les locaux vides ou peu encombrés prenant en compte la diffusion du son par les parois, d'autre part l'approche expérimentale qui a été faite du paramètre d'entrée décrivant la diffusion. 
Le modèle est basé sur une méthode classique de tirage de rayons, mais à l'issue de l'impact du rayon sur la paroi, seule une partie de l'énergie réfléchie est renvoyée dans la direction spéculaire. L'autre partie de l'énergie est distribuée de manière identique dans toutes les directions, simulant ainsi la diffusion. Pour ce faire, le point d'impact $\mathbf{M}$ du rayon sur la paroi est mémorisé ainsi que l'énergie restante $\mathrm{Er}$, c'est-à-dire l'énergie non absorbée et non réfléchie dans la direction spéculaire. On crée ensuite une source secondaire au point $M$ à laquelle on associe l'énergie $E r$.

Compte tenu du principe utilisé, le nombre de rayons devient rapidement très important, conduisant à des temps de calcul extrêmement longs̀. Nous avons donc été conduits à optimiser les paramètres de calcul (nombre de rayons secondaires créés à chaque réflexion, numéro d'ordre de suivi de la diffusion,...) afin de réduire les temps de calcul tout en conservant une précision suffisante.

On constate de manière générale que si les nombres de rayons primaires et secondaires (respectivement $\mathrm{Np}$ et $\mathrm{Ns}$ ) sont suffisamment importants, les conséquences d'un suivi de la diffusion aux ordres de réflexion supérieurs à un sont les suivantes :

- Aucune sur le niveau sonore,

- lissage de la courbe de décroissance temporelle, sans modification de sa pente. On remarque aussi que ce lissage peut être obtenu par augmentation de $\mathrm{Np}$ et Ns.

Le choix de Np et Ns dépend des coefficients d'absorption et de diffusion des parois du local ainsi que de la distance source-récepteur. Il apparait ainsi, que le suivi de la diffusion n'est pas nécessaire au delà du premier ordre de réflexion.

Le coefficient de diffusion $d$ qui représente la part de l'énergie diffusée après chaque réflexion, varie de 0 à 1 . Lorsque $d=0$, la paroi a un comportement parfaitement spéculaire, alors que dans le cas où $d=1$, toute l'énergie réfléchie est diffusée.

Nous avons réalisé des mesures de décroissances spatiales dans plusieurs halls industriels et modélisé ces locaux. Les mesures sont réalisées par bandes d'octave (250 à $4000 \mathrm{~Hz})$ en s'éloignant d'une source sonore de référence. A titre d'exemple, les graphes ci-après présentent les résultats obtenus à $1000 \mathrm{~Hz}$ dans un local de grande dimension et peu encombré. On compare les valeurs mesurées aux résultats de calculs effectués pour différentes valeurs des coefficients d'absorption et de diffusion.

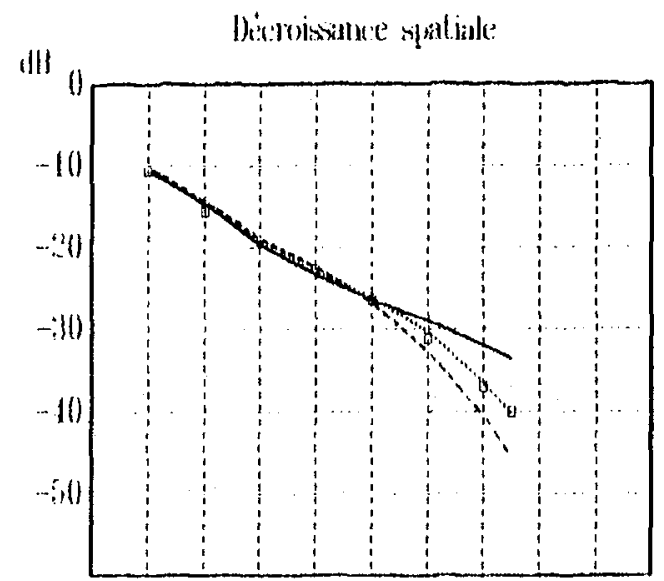

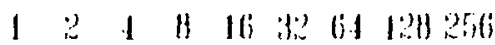

- calcul $d=0 \quad a=0.40$

.......... calcul $d=0.70 \quad a=0.15$

....... calcul $d=1 \quad a=0.15$

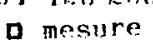

o mesure

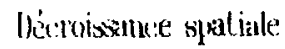

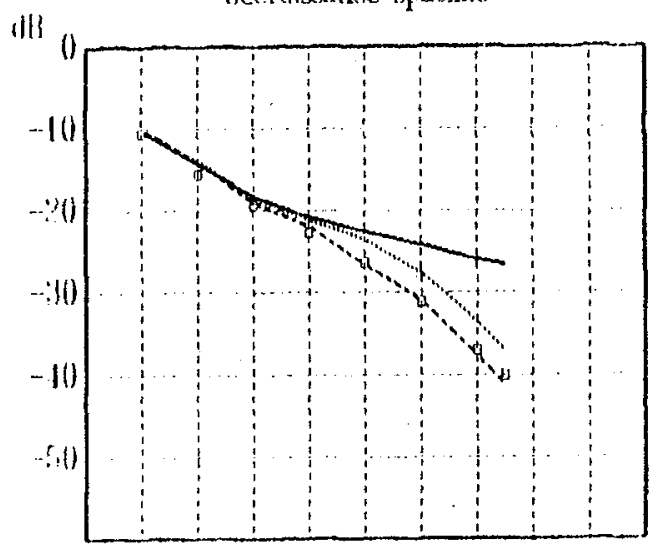

III

- culcul $d=0 \quad a=0.05$

a........calcul $d=0.70 \quad a=0.05$

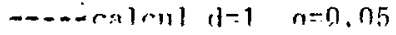

il 
L'axe des abcisses représente la distance source-récepteur, et l'axe des ordonnées la différence en $\mathrm{dB}$ entre le niveau de pression mesuré ou calculé et le niveau de puissance de la source.

On remarque que, même si le coefficient d'absorption choisi pour les parois du local est très sur-évalué, lorsque la réflexion est totalement spéculaire $(\mathrm{d}=0)$, le calcul ne permet pas d'obtenir d'accord avec la mesure dans la partie révèrbérée du champ. Ceci confirme la nécessité de prendre en compte l'influence de la diffusion. Ainsi, lorsque la réflexion est complètement diffuse $(\mathrm{d}=1)$, il est possible d'obtenir un très bon accord entre mesure et calcul sur toute la courbe ; cependant dans ce cas, il faut sous-évaluer le coefficient d'absorption des parois (alpha $=0.05$ ), puisque dans le local étudié, les parois possèdent un coefficient d'absorption moyen très classique, de l'ordre de 0.15 à $1000 \mathrm{~Hz}$. Lorsque l'on respecte cette valeur d'absorption, seul le calcul intermédiaire $(\mathrm{d}=0.7)$ permet alors d'obtenir l'accord mesure-calcul. Les mêmes constatations ont été réalisées dans les différents locaux modélisés.

Il apparait ainsi qu'une bonne connaissance du coefficient de diffusion à utiliser dans les calculs est nécessaire. Dans ce but, nous avons développé une méthode permettant de mesurer le coefficient de diffusion associé à un élément de surface. La méthode, dérivée des travaux de Vorländer sur la diffusion [4], est basée sur le calcul du coefficient d'intercorrelation entre deux réponses impulsionnelles. La première réponse est mesurée dans un local vide, la deuxième est mesurée dans le même local à l'intérieur duquel on a ajouté l'échantillon de surface à caractériser. Si les positions de la source et du microphone restent inchangées et que l'unique modification du local entre les deux mesures consiste en l'apport de l'échantillon à mesurer, le coefficient d'intercorrélation $\mathbf{k}$ entre les deux réponses impulsionnelles correspond au rapport de l'énergie réfléchie spéculairement sur l'énergie totale réfléchie. En effet dans ce cas, seule la part de l'énergie diffusée par l'échantillon est décorrélée par rapport à l'énergie réfléchie dans la direction spéculaire. Le calcul de $\mathrm{k}$ est réalisé sur la partie réverbérée des réponses impulsionnelles. On choisit de positionner source et microphone de telle sorte qu'il soit possible de distinguer l'onde directe des premières réflexions. On exprime ensuite le coefficient de diffusion $d$ en fonction du coefficient d'intercorrélation, des coefficients d'absorption et des surfaces du local de test et de l'échantillon. Nous avons réalisé un grand nombre de mesures sur différents types de structures.

La méthode s'avère fiable. En effet, les écarts types obtenus pour le coefficient $d$, calculés à partir des résultats sur dix mesures en moyenne, sont de l'ordre de 0.01 . Il faut noter que pour obtenir de tels résultats, de grandes précautions doivent être prises quant à la stabilité de la source et du microphone, en particulier pour les mesures à haute fréquence $(2000$ et $4000 \mathrm{~Hz}$ ). En effet, à ces fréquences, le moindre déplacement de la source ou du microphone conduit à une décorrélation entre les signaux et donc à une surévaluation de la diffusion. 
Nous avons ensuite, par une méthode statistique, tenté d'établir une relation entre les caractéristiques des parois (dimensions caractéristiques des objets diffusants, densité des objets,...) et le coefficient de diffusion. Bien que le nombre de mesures réalisés à l'heure actuelle soit trop faible pour être certains de la validité des lois énoncées, il semble possible dès à présent de regrouper les différents types de parois rencontrées en quatre grandes familles décrivant leur comportement en réflexion, à savoir : Parois peu diffusantes, moyennement diffusantes, diffusantes et très diffusantes. Cette approche macroscopique semble s'imposer dans un premier temps, compte tenu du fait qu'il n'existe pas pour linstant de technique permettant de caractériser la diffusion des parois in situ.

Les résultats obtenus jusqu'à présent permettent de valider le modèle ainsi que les paramètre d'entrées. La principale difficulté reste l'établissement de relations entre les échantillons de surfaces caractérisés et les parois réelles rencontrées dans les locaux. Notre technique de mesure de la diffusion ne peut être considérée que comme une première approche de ce coefficient. En effet, la densité et les dimensions caractéristiques des obstacles ne constituent qu'une partie des paramètres descriptifs de la paroi. On peut supposer par exemple, qu'une surface lisse et plane mais à réaction non localisée peut avoir un comportement légèrement diffusant. Dans ce cas, il semble que seule une mesure in situ peut permettre l'appréhension exacte du comportement de la paroi. Une telle technique n'est pas disponible aujourd'hui et serait probablement compliquée à mettre en oeuvre quand on connait les contraintes liées aux mesures in situ, en particulier dans les locaux industriels.

\section{Références :}

- [1] Sénat C., Zuliani P., Guilhot J.P., Gamba R., Un modèle de prévision des niveaux de pression dans les locaux vides et encombrés reposant sur l'hypothèse de réflexion diffuse en parois, $\mathrm{J}$. Acoustique, 1991, pp. 335-350.

- [2] Kuttruff H., Room Acoustics (Applied Science Publishers, 1973), pp. 96-103.

-[3] Hodgson M., J. Acoust. Soc. Am. 89 (2), February 1991, pp. 765-771.

-[4] Vorländer.M., Untersuchungen zur Leistungsfähigkeit des raumakustischen Schallteichenmodells, Thèse de Doctorat, Février 1989, pp. 126-133. 